\title{
Research on Photo Neutron Dose to Patient by $(n, p)$ Reaction from a 15 MV Linear Accelerator
}

\author{
K.R. Rajesh, R. Ganapathi Raman
}

\begin{abstract}
Introduction: Photoneutron produced during radiotherapy treatment was measured and the possibility for the radiation yield by the $(n, p)$ reaction within the body was calculated.

Materials and Methods: Photoneutron spectrum was measured using Columbia Resin (CR 39) film from a Siemens Primus Plus Linear accelerator and the possibility for the radiation yield by the $(n, p)$ reaction within the body was calculated during radiotherapy treatment.

Results--- The photoneutron spectrum was measured with $C R$ 39 SSNTD and automatic track counting software. The interaction cross section for the $(n, p)$ reaction for each energy and element were determined and radiation yield was calculated.For hydrogen, there is no $(n, p)$ reaction. The $(n, p)$ reaction is absent in C12for neutron of energy below $13.5 \mathrm{MeV}$. Oxygen has $(n, p)$ reaction above $10 \mathrm{MeV}$. Nitrogen has a low threshold and there is no threshold for potassium and calcium.

Conclusion--- From the result, it is clear that $(n, p)$ reactions are significant and even though the dose from these reactions is low, the impact will be severe due to the high LET nature of proton. The data can be used to minimize the photoneutron production during the radiotherapy treatment in the future. The outcome of the work indicates the importance of photoneutron transport and dose deposition to be included in the treatment planning algorithm.
\end{abstract}

Keywords--- Columbia Resin-39 Detector, Medical Linear Accelerator, Photoneutron Tracks.

\section{INTRODUCTION}

It is well accepted that medical linear accelerators produce photo-neutrons having energy range from thermal to fast neutron energies [1]. The peak energy of the neutrons producing in a particular energy mode is expressed as $\mathrm{E}_{\max }-$ $E_{n}$, where $E_{\max }$ is the peak photon energy and $E_{n}$ is the neutron separation energy of the target. The output neutron spectrum is dependent upon the relative yield of the neutron producing channels, the energy of the photon projectile and resonances in the channels. These unwanted neutrons are contaminating the treatment photon field and producing an excess dose to the patient [2]. It is well understood that the radiobiological effectiveness substantially higher than photons [3]. Therefore a small number of neutrons can lead to a non-negligible dose component The isotope formed during the (n, p) reactions are radioactive. Among these, some are short-lived but not all. These radioactive isotopes decay by beta and gamma emission and expose further dose to the patient. The chances of potential hazard by the longlived isotope to the personnel cannot be neglected [4].The

Revised Manuscript Received on July 10, 2019.

K.R. Rajesh, Caritas Hospital, Kottayam, Kerala. India

Department of Physics, Noorul Islam Centre for Higher Education, Kumaracoil, Tamil Nadu. India

R. Ganapathi Raman, Department of Physics, Noorul Islam Centre for Higher Education, Kumaracoil, Tamil Nadu, India.(e-mail: ganapathiraman83@gmail.com) electron contamination associated with photons also causes severe effects [5].

Present neutron dosimetry trends are based on the LiFTLD chips, CR-39 Bulk etch based dosimetry and BF3 counters which are strictly relative dosimetry standards $[6$, 7]. This requires a standard neuron field is present. In any of these relative dosimetry trends, the measurement will be accurate only if the neutron spectrum is just alike to the calibration spectrum. Because of the difference in neutron spectra generated from each machine, a spectroscopy-based method is required for the absolute neutron dosimetry. But the active neutron spectrometers like NE213- Liquid Scintillator, Anthracene based plastic scintillators etc. are sensitive to photons which will suppress the neutron spectroscopic information. Therefore it demands a spectrometry method which is irrespective of photons. This leads to the use of a passive neutron spectroscopy method based on SSNTD and from the measured spectrum the dose is measured through the well-accepted kerma method $[8,9]$.

\section{MATERIALS AND METHODS}

The neutron energy spectrum was measured using CR-39 SSNTD. The CR39 SSNTD used in this work was a $250 \mu \mathrm{m}$ thick film and density $1.3 \mathrm{~g} / \mathrm{cc}[10]$. The film was cut into a dimension of $1 \mathrm{~cm}^{*} 1 \mathrm{~cm}$ using a diamond cutter. The film was given an identification tag for future reference. The film was placed on the patient $5 \mathrm{~cm}$ top from the anterior field edge (Figure 1). The patient was treated with 3DCRT with box field (four fields) technique with 15MV energy used to deliver 200cGy. Thermoplastic material was used as immobilization device.

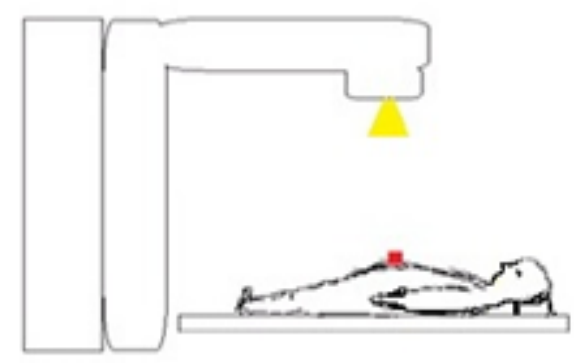

Figure 1: Irradiation set up

The exposed SSNTD was etched in $6 \mathrm{~N} \mathrm{NaOH}$, for $6 \mathrm{Hrs}$ at $60^{\circ} \mathrm{C}$ constant temperature. The image of the film was captured with a microscopein 40X magnification (Figure 2). This is then analyzed using the TRIAC-2 code, major axis; minor axis and track angle were obtained[11].

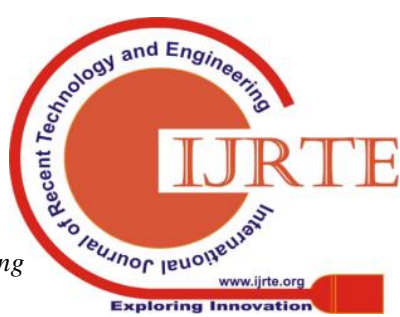




\section{RESEARCH ON PHOTO NEUTRON DOSE TO PATIENT BY (N, P) REACTION FROM A 15 MV LINEAR ACCELERATOR}

Proton energy was deduced form energy-diameter calibration and the neutron energy was calculated, with the recoil angle deduced for each track $[12,13]$. The energy distribution was made into successive bins of $250 \mathrm{KeV}$ and the numbers of tracks in each bin were tabulated. The number of neutrons corresponds to each bin were calculated with the yield obtained, number of hydrogen targets per $\mathrm{cm}^{2}$ in the CR-39 film and n-p elastic scattering cross section. The energy versus count of the neutron is plotted using GNU plot(Figure 3).For a single neutron energy, type of target atom, and kind of interaction, the kerma that results from a neutron fluence $\varphi\left(\mathrm{cm}^{-}\right)$at a point in a medium is given by

$$
\mathbf{K}=1.602 \times 10^{\wedge}(-8)^{*}(\varphi)^{*}(\sigma) *\left(N_{T}\right) * m^{\wedge}(-1) *\left(E_{t r}\right) G y
$$

where $\boldsymbol{\sigma}$ is the interaction cross section in $\mathrm{cm}^{\prime} / /$ target atom), $N$, is the number of target atoms in the irradiated sample, $\mathbf{m}$ is the sample mass in grams, and Etris the total kinetic energy $(\mathrm{MeV})$ given to charged particles per interaction. $\mathrm{K}$ is thus given in rad (or centi grays), and its value is equal to the absorbed dose $\mathrm{D}$ at the same point under the usual CPE conditions.

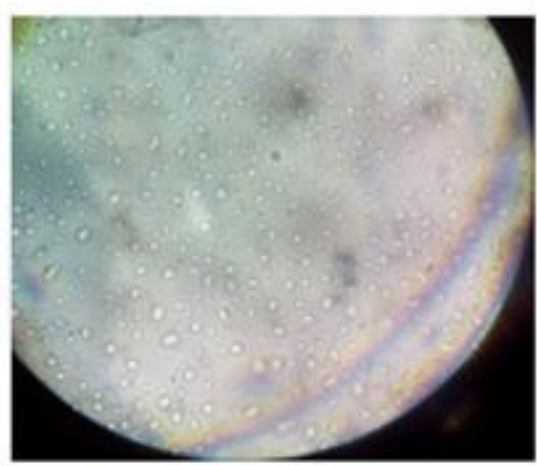

Figure 2: Erched CR 39 Film

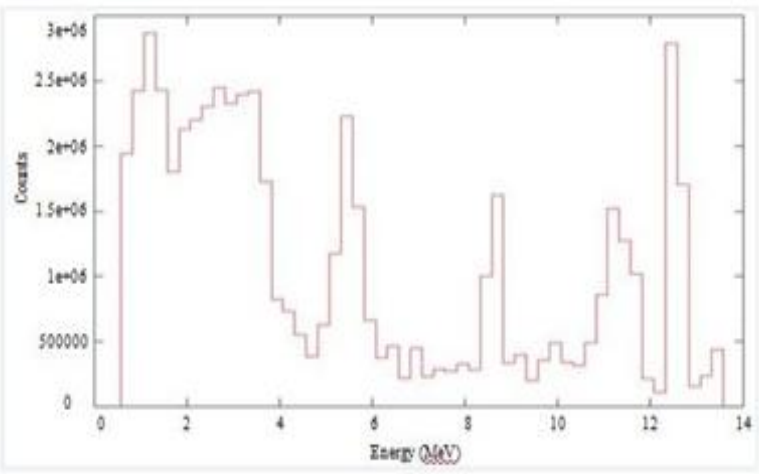

Figure 3: Photoneutron Spectrum

In our procedure, we have averaged the unit volume throughout the body and taken as per kilogram so the averaging factor taken in above equation becomes reduced to

$$
\mathbf{K}=(\varphi) *(\sigma) *\left(\mathbf{N}_{\mathrm{T}}\right) *\left(\mathbf{E}_{\mathrm{tr}}\right) \mathbf{G y}
$$

The ICRU composition for muscle has been assumed in most cases for neutron-dose calculations (see ICRU, 1977), lumping the $1.1 \%$ of "other" minor elements together with oxygen to make a simple four-element $(\mathrm{H}, \mathrm{O}, \mathrm{C}, \mathrm{N})$ composition.

The interaction cross section values were obtained for each energy bin and element for the $(n, p)$ reaction and the radiation yield were calculated using the above equation [14, 15]. The total radiation yield was calculated by taking all the contributions from different elements in the body (Table 1).
Table 1: Total Radiation Yield

\begin{tabular}{|c|c|c|c|c|c|c|}
\hline \multicolumn{5}{|c|}{ RADIATION YIELD FOR (n,p) REACTIONS } & \multirow{2}{*}{$\begin{array}{c}\text { TOTAL } \\
\text { (Gy) }\end{array}$} \\
\cline { 1 - 5 } $\mathrm{C}^{12}$ & $\mathrm{~N}^{14}$ & $\mathrm{O}^{16}$ & $\mathrm{p}^{30}$ & $\mathrm{Ca}^{40}$ & $\mathrm{~K}^{39}$ & \\
\hline 0 & $\begin{array}{c}1.403 \mathrm{x} \\
10^{-6}\end{array}$ & $\begin{array}{c}1.526 \mathrm{x} \\
10^{-6}\end{array}$ & $\begin{array}{c}3.79 \mathrm{x} \\
10^{-6}\end{array}$ & $\begin{array}{c}2.104 \mathrm{x} \\
10^{-6}\end{array}$ & $\begin{array}{c}4.126 \mathrm{x} \\
10^{-7}\end{array}$ & $\begin{array}{c}9.23 \mathrm{x} \\
10^{-6}\end{array}$ \\
& & & & & \\
\hline
\end{tabular}

\section{RESULTS}

The photoneutrons are recorded using SSNTD films (CR39 films).The energy versus count of the neutron is plotted using GNU plot. For 15MV photon, we got neutrons of energy up to $13 \mathrm{MeV}$. From the graph, it is clear that neutron of lower energy $(2 \mathrm{MeV}-4 \mathrm{MeV})$ is most abuntant. Attention was given to study about $(n, p)$ reaction within the patient body. Patient body is composed of primarily carbon, hydrogen, oxygen, nitrogen, potassium phosphorus etc. Among this most of the elements have a certain threshold for $(n, p)$ reaction. For hydrogen, there is no $(n, p)$ reaction. The $(n, p)$ reaction is absent in $C^{12}$ for neutron of energy below $13.5 \mathrm{MeV}$. Oxygen has $(\mathrm{n}, \mathrm{p})$ reaction above $10 \mathrm{MeV}$. Nitrogen has alow threshold and there is no threshold for potassium and calcium. The interaction cross section for the $(n, p)$ reaction for each energy and element was determined and radiation yield from each element were calculated.Similarly the radiation yield was calculated for each element and the total was calculated.

\section{DISCUSSION}

The work is focused mainly on the $(n, p)$ reactions that is occurring during the radiotherapy treatment. There may be also the possibility of $(n, \infty)$ reactions which can also deposit dose in the patient. The Monte Carlo simulations can be used for summarising all the reactions that can take place during the radiotherapy treatment $[16,17,18,19,20]$. The present work initially accounts the photoneutron spectrum using CR 39 SSNTD, and then calculating the dose deposited by the (n, p) reactions with the help of interaction crossection data and kerma equations. The work can be extended to include thermal neutrons and out off field photoneutron dosimetry which may help a lot in the future in the design of dose calculation algorithms.

\section{CONCLUSION}

The present work gives an insight into the photoneutron dose deposition by $(\mathrm{n}, \mathrm{p})$ reaction, which is one of the mechanisms by which photoneutron deposit dose inside the patient body. The dose deposition by the photoneutrons far away from the treatment site may cause secondary cancer induction in the future.

\section{Published By:}


The different reaction channels responsible for photoneutron production can be identified from the spectrum generated using CR 39 SSNTD. The data can be used to minimize the photoneutron production during the radiotherapy treatment in the future. The outcomes of the work indicate the importance of photoneutron transport and dose deposition to be included in the treatment planning algorithm.

\section{ACKNOWLEDGEMENT}

The authors are grateful for the invaluable support from Dept.of Physics, Calicut University and Dept. Radiation Oncology, Caritas hospital.

\section{REFERENCES}

1. NCRP REPORT No. 79, Neutron contamination from medical electron accelerators (1984).

2. F. Difilippo et al Contamination dose from photoneutron processes in bodily tissues during therapeutic radiation delivery.

3. Hosseini Aghdam MR, Baghani HR, Mahdavi SR, Aghamiri SMR. Cancer Risk Assessment due to Accidental Exposure inside Neutron Laboratories using BEIR VII Model. Iran J Med Phys 2018; 15: 251-255.

4. Thomadsen, Bruce, et al. "Potential Hazard Due to Induced Radioactivity Secondary to Radiotherapy: The Report of Task Group 136 of the American Association of Physicists in Medicine.

5. Seyed Mostafa Ghavami1, Hosein Ghias. Estimation of Secondary Skin Cancer Risk Due To Electron Contamination in 18-MV LINAC-Based Prostate Radiotherapy. Iran J Med Phys Vol. 13, No. 4, December 2016, 236-249.

6. Yücel, Haluk, İbrahim Çobanbaş, AsumanKolbaş1, AlptuğÖzerYüksel, and Vildan Kaya. "Measurement of Photo-Neutron Dose from an 18-MV Medical Linac Using a Foil Activation Method in View of Radiation Protection of Patients."AAPM American Association of Physicist in Medicine REPORT No 19, Neutron measurements.

7. El-Sersy, A. R., S. A. Eman, and N. E. Khaled. "Fast neutron spectroscopy using CR-39 track detectors."

8. T.Tsuruta et. Al. Fast Neutron Dosimetry with CR-39 Plastic Plate.

9. N.E.Ipe et al "A comparison of the neutron response of cr-39 made by different manufacturers".

10. Patiris, K. Blekas, K.G. Ioannides. "TRIAC: A code for track measurements using image analysis tools". Nuclear Instruments and Methods in Physics Research 2006: B 244:392-96.

11. MatiullahTufail M, Ahmad N, Khan G, Manzoor S. and Khan, H.. Some investigations on the response of the CR-39 detector to protons, deuterons and alpha particles. International Journal of Radiation Applications and Instrumentation. Part D. Nuclear Tracks and Radiation Measurements, (1988) 15(1-4), pp.137-140.

12. Antony Joseph, (1993). SOME NUCLEAR STUDIES USING CR-39 TRACK DETECTOR. Ph.D. Dissertation. The University of Calicut.

13. J.A. Auxier and M.D. Brown " Neutron cross sections and reaction product for $\mathrm{H}, \mathrm{C}, \mathrm{N}$ and $\mathrm{O}$ for energy range from thermal to $15 \mathrm{MeV}$.

14. Masahide Harada et al Evaluation of Neutron Cross Sections of Carbon-12 for Energies up to $80 \mathrm{MeV}$.

15. T Siji Cyriac, R Ganapathi Raman, M M Musthafa, A K Bakshi, Rupali Pal, Sandipan Dawn, K Abdul Haneefa, T Palani Selvam and D Datta "Study on the measurement of photo-neutron for $15 \mathrm{MV}$ photon beam from Medical
Linear accelerator under different irradiation geometries using passive detectors" Journal of Cancer Research and Therapeutics 2016 (12) 2 1060-1064 doi: 10.4103/09731482.183187 ISSN, 0973-1482

16. Abdul Haneefa, K., T. Siji Cyriac, M. M. Musthafa, R. Ganapathi Raman, V. T. Hridya, A. Siddhartha, and K. K. Shakir."FLUKA Monte Carlo for basic dosimetric studies of dual energy medical linear accelerator." Journal of Radiotherapy, Article ID 343979, http://dx.doi.org/10.1155/2014/343979 (2014).

17. Siji Cyriac, M.M. Musthafa, R. Ganapathi Raman, Abdul Haneefa K, Saju Bhasi "Out-of-Field Photon Dosimetry Studies in 3-D Conformal and Intensity Modulated Radiation Therapy Treatment for Prostate Cancer"International Journal of Radiation Research, (2015) 13(2) 127-134. DOI: 10.7508/ijrr.2015.02.002

18. Cyriac Siji, MM Musthafa, R. Ganapathi raman, Kummali Abdul Haneefa, Hridya VT "Pretreatment patient specific quality assurance and gamma index variation study in gantry dependent EPID positions for IMRT prostate treatments." Journal of Radiotherapy Article $\quad$ ID 325057 (2014). http://dx.doi.org/10.1155/2014/325057

19. Abdul Haneefa, K., Shakir, K. K., Siddhartha, A., Cyriac, T. S., Musthafa, M. M., \& Ganapthi Raman, R "Dosimetric studies of mixed energy intensity modulated radiation therapy for prostate cancer treatments." Journal of Radiotherapy, Article ID 760206 (2014). 